\title{
SP110 as a novel susceptibility gene for Mycobacterium avium subspecies paratuberculosis infection in cattle
}

\author{
O. Ruiz-Larrañaga, ${ }^{\star}$ J. M. Garrido, $†$ M. Iriondo, ${ }^{\star}$ C. Manzano, ${ }^{*}$ E. Molina, $\dagger$ I. Montes, ${ }^{\star}$ P. Vazquez, $\dagger$ \\ A. P. Koets, $¥$ V. P. M. G. Rutten,§\# R. A. Juste, $†$ and A. Estonba*1 \\ *Genetics, Physical Anthropology, and Animal Physiology Department, University of the Basque Country, Sarriena s/n, 48940 Leioa, Bizkaia, \\ Spain \\ †Animal Health Department, NEIKER-Teknalia, Berreaga 1, 48160 Derio, Bizkaia, Spain \\ ‡Department of Farm Animal Health and \\ SInstitute of Infectious Diseases and Immunology, Department of Immunology, Faculty of Veterinary Medicine, Utrecht University, \\ Utrecht, the Netherlands \\ \#Department of Veterinary Tropical Diseases, Faculty of Veterinary Science, University of Pretoria, Private Bag X04, Onderstepoort 0110, \\ Republic of South Africa
}

\section{ABSTRACT}

The intracellular pathogen resistance 1 (Ipr1) gene has been reported to play a role in mediating innate immunity in a mouse model of Mycobacterium tuberculosis infection, and polymorphisms of its human ortholog, SP110 nuclear body protein, have been suggested to be associated with tuberculosis. Thus, the bovine SP110 gene was considered to be a promising candidate for a genetic association study of bovine paratuberculosis, or Johne's disease, a chronic granulomatous enteritis caused by Mycobacterium avium ssp. paratuberculosis (MAP). Initially, single nucleotide polymorphisms (SNP) within the bovine SP110 gene were identified, and subsequently a population-based genetic association study was carried out. Seventeen new SNP along the SP110 gene were identified in Holstein-Friesian cattle, and 6 more were compiled from public databases. A total of 14 SNP were included in the association study of 2 independent populations. The SNP c.587A $>\mathrm{G}$ was found to be significantly associated with MAP infection, with the major allele A appearing to confer greater disease susceptibility in one of the analyzed populations. In addition, 2 haplotypes containing this SNP were also found to be associated with infection in the same population. The SNP c.587A $>\mathrm{G}$ is a nonsynonymous mutation that causes an amino acid change in codon 196 from asparagine to serine. In silico analyses point to $\mathrm{SNP}$ c.587A $>\mathrm{G}$ as a putative causal variant for susceptibility to MAP infection. The elucidation of the precise mechanism by which this SNP can exert its effect in the protein and, as a result, in the risk of infection, requires future functional analyses. Likewise, the absence of genetic association in

Received April 14, 2010

Accepted July 30, 2010

${ }^{1}$ Corresponding author: andone.estonba@ehu.es one of the analyzed populations renders it necessary to carry out this study in other independent populations, with the aim of substantiating the repeatability of the present results. Nevertheless, the present results deepen our understanding of the genetic basis of susceptibility and resistance mechanisms related to MAP infection in cattle and, in turn, constitute a step forward toward the implementation of marker-assisted selection in breeding programs aimed at controlling paratuberculosis.

Key words: SP110, paratuberculosis, single nucleotide polymorphism, marker-assisted selection

\section{INTRODUCTION}

The potential of marker-assisted selection to enhance resistance to infectious diseases in dairy cattle has been the motivation behind numerous studies aimed at identifying genes that confer susceptibility to this kind of disease (Schulman et al., 2004; Sharma et al., 2006; Juliarena et al., 2008). This is also the case with studies related to bovine paratuberculosis, or Johne's disease, a chronic granulomatous enteritis caused by Mycobacterium avium ssp. paratuberculosis (MAP), which has a profound economic effect on the dairy industry. Recently, different innate immune genes such as solute carrier family 11 member 1 (SLC11A1), nucleotide-binding oligomerization domain 2 (NOD2), toll-like receptor 1 (TLR1), toll-like receptor 2 (TLR2), and toll-like receptor 4 (TLR4) have been found to be associated with susceptibility and resistance to MAP infection (Koets et al., 2010; Mucha et al., 2009; Pinedo et al., 2009a,b; Ruiz-Larrañaga et al., 2010a,b). Nevertheless, similar other complex diseases, paratuberculosis is thought to be a polygenic disease, i.e., controlled by a large number of genes that interact with one another and with environmental factors. Thus, the identification of new susceptibility genes will increase our understanding of the molecular basis of susceptibility and resistance to 
MAP infection; the candidate gene approach is a powerful method to achieve this goal.

The intracellular pathogen resistance 1 (Ipr1) gene is involved in triggering innate immune responses to multiple intracellular pathogens. In a murine model of Mycobacterium tuberculosis infection, Ipr1 expression was upregulated in murine macrophages resistant to $M$. tuberculosis but was absent in susceptible ones; moreover, expression of an Ipr1 transgene construct in macrophages from susceptible animals resulted in the control of mycobacterial replication and eventually induced the death of infected cells (Pan et al., 2005). Thus, Ipr1 appears to play an important role in the control of $M$. tuberculosis within host macrophages. The human Ipr1 protein ortholog, SP110 nuclear body protein $(\boldsymbol{S P 1 1 0})$, has been identified as a complement of the nuclear body multiprotein complex, and although its function is not well understood, it is thought to play a role in the differentiation of myeloid cells and also act as a transcriptional coactivator (Bloch et al., 2000). Three SNP polymorphisms of the SP110 gene have been found to be associated with tuberculosis susceptibility in humans (Tosh et al., 2006). For these reasons, the bovine SP110 gene, localized on BTA2, was selected as a promising candidate gene for MAP infection susceptibility in cattle. To date, no studies of the bovine SP110 gene have been completed, and polymorphisms of this gene have only rarely been reported in SNP databases. Therefore, this work was undertaken with a double objective: first, to extensively characterize the variability of the bovine SP110 gene in the European HolsteinFriesian (HF) breed by means of the identification of new SNP along the gene locus including exons, introns, untranslated regions, and a part of the promoter, and second, to perform a population-based genetic association study to test the putative relation between these SNP and MAP infection.

\section{MATERIALS AND METHODS SNP Discovery and Selection}

A comparative sequencing strategy of $30 \mathrm{HF}$ animals was followed for the detection of new SNP in the bovine SP110 gene. Thirteen fragments were amplified and sequenced, which harbored 13 of the 16 exons that make up the gene, their adjacent intronic regions, untranslated regions, and $300 \mathrm{bp}$ from the promoter of the gene likely to contain regulatory elements. Primers for amplification and sequencing (supplemental Table S1, www.journalofdairyscience.org/) were designed based on the SP110 genomic DNA sequence from the NCBI Gene database using the Primer3 program (http://frodo.wi.mit.edu/primer3/). Conventional or touchdown
PCR was carried out using a GeneAmp 9700 PCR System (Applied Biosystems, Foster City, CA) with 10 to $15 \mathrm{ng}$ of genomic DNA as a template in a final volume of $25 \mu \mathrm{L}$. Sequencing reactions were performed using BigDyeR Terminator 3.1 Cycle Sequencing Kit chemistry and resolved with capillary electrophoresis on the Genetic Analyzer 3130xl sequencer (Applied Biosystems). The SNP were identified by alignment of the sequences obtained from each individual and their comparison with the LOC515204 reference sequence from the NCBI database, using SeqScape 2.5 software (Applied Biosystems). Detected SNP were designated according to the nomenclature for the description of sequence variations by the Human Genome Variation Society (http://www.hgvs.org/mutnomen/).

As a complementary strategy for SNP discovery, polymorphisms in the bovine SP110 gene that were found in NCBI dbSNP, Ensembl, and IBISS (Hawken et al., 2004) databases were annotated. Single nucleotide polymorphism selection for subsequent genotyping was performed following Custom TaqMan Genomic Assays Protocol Submission Guidelines (Applied Biosystems, 2006) criteria and methodological compatibilities in the design of probes.

\section{Population-Based Genetic Association Analysis}

The population for the genetic association study was made up of $766 \mathrm{HF}$ adult cows (older than $2 \mathrm{yr}$ ) from 2 independent subpopulations. Cases and controls were identified from a Spanish population of $355 \mathrm{HF}$ animals belonging to 30 herds (HFS). All these samples were received at the Neiker-Teknalia technological center (Derio, Spain) for confirmation of a clinical suspicion of paratuberculosis in the herd and had no further followup. Animals were classified as infected (cases) if they tested positive in fecal culture or blood serum ELISA tests; in a total of 122 cases, 95 animals were ELISA positive, 10 animals were culture positive, and 17 animals were positive for both diagnostic tests. Animals with negative results for both tests were considered healthy (controls; $n=233$ ). Independently, cases and controls were identified in a cohort of $411 \mathrm{HF}$ animals from 8 Dutch dairy farms (HFN). These animals were sampled on at least 4 occasions, and fecal culture and blood serum ELISA tests were performed. A case was defined as having at least one fecal culture positive test result $(\mathrm{n}=182)$. Of these infected animals, 46 were positive for both tests. Animals tested at least 4 times that were negative to all tests were considered controls $(\mathrm{n}=229)$. A description of the diagnostic tests employed is reported elsewhere (Ruiz-Larrañaga et al., 2010a). 
All herds of origin of the animals had been exposed to the mycobacteria, and the average infection prevalence was $6 \%$ in Spain and $32.2 \%$ in the Netherlands. This study was carried out on the assumption that MAP is widespread in European ruminant populations and that, once MAP infection is present in a herd, all animals are potentially exposed to it. To test for population stratification, 17 neutral microsatellites were genotyped in 100 unrelated cows, 50 from each subpopulation (25 infected and 25 healthy). Comparison of allelic frequencies between infected and healthy subgroups did not indicate any potential stratification for bias association analyses in both HFS and HFN subpopulations (all $P$ values were above 0.003, after Bonferroni correction).

Deoxyribonucleic acid was extracted from blood samples using a QIAamp Mini Kit (Qiagen, Hilden, Germany). Genotyping of selected bovine SP110 SNP was performed by TaqManOpenArray technology (Applied Biosystems). For each array, 2 negative controls and a duplicated sample, as a positive control, were included. Alleles of individuals for each SNP were assigned using BioTrove OpenArray SNP Genotyping Analysis software 1.0.3 (Applied Biosystems). The SNP were checked by Haploview v4.1 statistical software (Barrett et al., 2005; http://www.broadinstitute. org/mpg/haploview) for 2 quality control parameters: call rate (minimum of $75 \%$ required) and conformity of genotype proportions to Hardy-Weinberg equilibrium in healthy individuals. Individual animals with low call rates $(<50 \%)$ were excluded from the subsequent association analyses.

The latter software was also used to establish linkage disequilibrium for each SNP pair by $\mathrm{r}^{2}$ and $\mathrm{D}^{\prime}$ parameters, to plot haplotype block structure by the confidence intervals method (Gabriel et al., 2002), and to calculate $P$-values for allelic and haplotype associations, comparing frequencies in infected versus healthy individuals. Analysis of the genotypic association was carried out using the SNPassoc library (González et al., 2007) from R v2.10.1 software ( R Development Core Team, 2008). R software offers the possibility to perform the statistical test for minor alleles under 5 different genetic models simultaneously: codominant, dominant, recessive, overdominant, and log-additive. Odds ratios and $P$-values for the likelihood ratio test of association were calculated for these genetic models. Results of genotypic association were also adjusted by herd with a view of considering its possible effect in the analyses. To correct for the occurrence of false positives (type I error), permutation procedures were performed in all association analyses (1,000,000 permutations). The permutation test corrects for multiple testing, resampling $\mathrm{N}$ times the total number of observations in a population, to build an empirical estimate of the null distribution from which the test statistic has been drawn. This approach takes into account the correlation between markers. Permutation correction is thus less conservative than the Bonferroni correction, but it is appropriate for independent tests with multiple markers (Camargo et al., 2008). Genetic differentiation analyses were performed with the GENEPOP v4.0 package (Rousset, 2008) to test for differences in allele frequencies of analyzed SNP between HFS and HFN subpopulations.

\section{In Silico Study of the Associated SNP}

Alignments of the predicted mRNA and protein sequences corresponding to the bovine SP110 gene with the orthologous sequences in human (Homo sapiens), chimpanzee (Pan troglodytes), mouse (Mus musculus), rat (Rattus norvegicus), and horse (Equus caballus) were performed with the ClustalW Multiple Alignment option from the BioEdit Sequence Alignment Editor (http://www.mbio.ncsu.edu/BioEdit/BioEdit.html) and MULTALIN software (Corpet, 1988), respectively. The ELM server (http://elm.eu.org/) was used to predict putative functional sites in the amino acid sequence of the SP110 protein. JUFO software (http://www. meilerlab.org/web/view.php) was applied to predict changes in the secondary structure of the protein.

\section{RESULTS}

\section{SNP Discovery and Selection}

Genomic DNA samples from $30 \mathrm{HF}$ cattle were screened for SNP polymorphisms in the SP110 gene by comparative sequencing. From 13 amplified fragments, whose lengths ranged from 423 to $763 \mathrm{bp}$, with an average of $604 \mathrm{bp}, 7,857 \mathrm{bp}$ of the $51,142 \mathrm{bp}$ of the gene were sequenced. This comparative sequence analysis revealed 18 SNP in the HF breed. Of these, 17 were novel, being described for the first time in the Bos taurus species, whereas the other corresponded to the rs41256732 SNP from the NCBI dbSNP database (supplemental Table S2, www.journalofdairyscience.org/). The SNP c.587A $>$ G and c.1769C $>$ T (rs41256732) were nonsynonymous SNP located in exon 5 and exon 14, respectively. These SNP lead to an Asn $\rightarrow$ Ser amino acid change at codon 196 (p.Asn196Ser) and a Pro $\rightarrow$ Leu change at position 590 (p.Pro590Leu), respectively. The other 16 SNP were intronic, with 9 of them being transitions and 7 being transversions. The SNP were not found in the promoter or untranslated regions.

From the NCBI dbSNP and IBISS databases, 5 additional SNP were annotated (supplemental Table S2). These SNP were localized outside the amplified frag- 


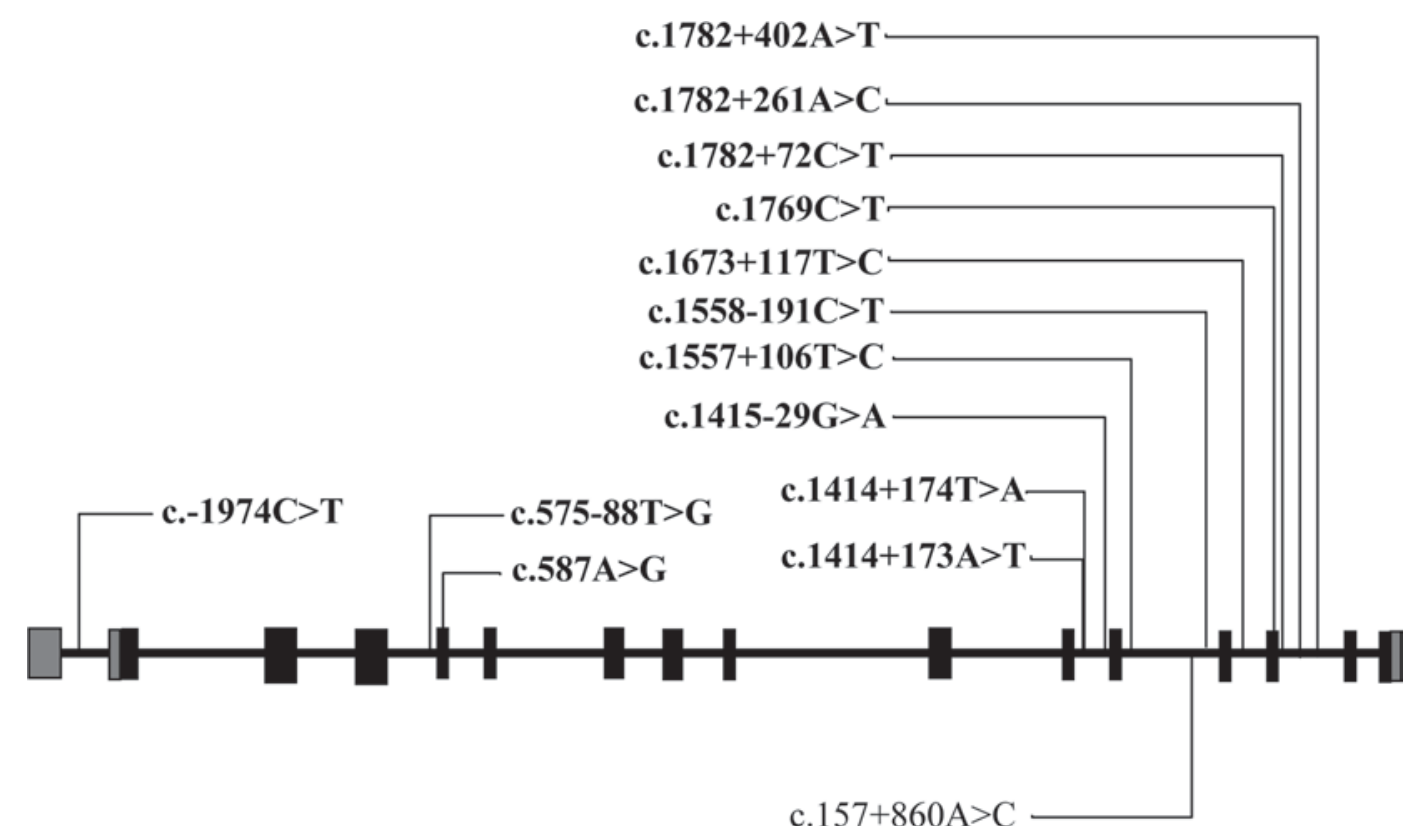

Figure 1. Genomic structure and analyzed polymorphisms of the bovine SP110 nuclear body protein gene. Black and gray boxes indicate exons and untranslated regions, respectively, and lines connecting them indicate introns. Polymorphic SNP in European Holstein-Friesian cattle are represented in bold.

ments and corresponded to intronic polymorphisms. In all, 23 SNP were compiled as candidates for the genotyping process. Finally, and according to Custom TaqMan Genomic Assays Protocol Submission Guidelines (Applied Biosystems, 2006) criteria and methodological compatibilities in the design of probes, 14 SNP were selected (Figure 1). All the newly identified SNP were submitted to GenBank under accession numbers ss184956656 to ss184956675.

\section{SNP Genotyping}

A total of 14 SNP were included in the genotyping of $766 \mathrm{HF}$ cattle (supplemental Table S3, www. journalofdairyscience.org/). All of these SNP were successfully genotyped on $90 \%$ or more of the samples and found in Hardy-Weinberg equilibrium in both the Spanish and the Dutch control subgroups $(P>0.001)$. Because of their low call rate $(<50 \%), 31$ individuals were excluded from genetic association analyses. The SNP c. $1557+860 \mathrm{~A}>\mathrm{C}(\mathrm{rs} 43322128)$ was found to be monomorphic in HFS and HFN populations. Among the polymorphic SNP, c. $1782+72 \mathrm{C}>\mathrm{T}$ and SNP c.$1974 \mathrm{C}>\mathrm{T}$ had a minor allele frequency $<0.05$ in HFS and $\mathrm{HFN}$, and in HFN, respectively.

\section{Genetic Association Analyses}

The exonic SNP c.587A $>$ G in HFS was found to be genetically associated with MAP infection (corrected
$P=2 \times 10^{-4}$, Table 1$)$; its major A allele occurred at a significantly greater frequency in infected animals than in healthy ones. Genotypic association analysis for SNP c.587A $>$ G in this subpopulation, adjusted by herd, resulted in the greatest significance value for the additive model of alleles $(P<0.008$ after correction by $1,000,000$ permutations). The minor $\mathrm{G}$ allele reduced the risk of MAP infection with a per-allele odds ratio of $0.36(95 \%$ CI $=0.22-0.59)$. In HFN, no SNP was found to be significantly associated with infection, although alleles from SNP c.587A $>$ G showed the same tendency as in HFS (nominal $P=0.031$, Table 1).

Linkage disequilibrium analyses between each SNP pair in the SP110 gene defined the same distribution of haplotype blocks in both HFS and HFN (Figure 2). The first haplotype block included SNP c.575-88T $>$ G, together with c.587A $>\mathrm{G}\left(\mathrm{D}^{\prime}=1.000\right.$ and $\mathrm{r}^{2}=0.111 \mathrm{in}$ HFS; $\mathrm{D}^{\prime}=1.000$ and $\mathrm{r}^{2}=0.172$ in HFN), for which 3 haplotypes, TA, GA, and TG, were detected in both HF populations (Table 2). The second haplotype block was composed of the following SNP: c. $1414+173 \mathrm{~A}>\mathrm{T}$, c. $1414+174 \mathrm{~T}>\mathrm{A}, \quad$ c. $1415-29 \mathrm{G}>\mathrm{A}, \quad$ c. $1557+106 \mathrm{~T}>\mathrm{C}$, c. $1558-191 \mathrm{C}>\mathrm{T}, \quad$ c. $1673+117 \mathrm{~T}>\mathrm{C}, \quad$ c. $1769 \mathrm{C}>\mathrm{T}$, c. $1782+261 \mathrm{~A}>\mathrm{C}$, and c. $1782+402 \mathrm{~A}>\mathrm{T}\left(\mathrm{D}^{\prime}=0.919\right.$ 1.000 and $r^{2}=0.172$ in HFS; $\mathrm{D}^{\prime}=0.972-1.000$ and $\mathrm{r}^{2}$ $=0.096-1.000$ in HFN), for which 8 and 9 haplotypes were observed in HFS and HFN, respectively. The SNP c. $-1974 \mathrm{C}>\mathrm{T}$ remained outside both blocks. Association analysis in HFS indicated that the TA haplotype from 
Table 1. Chi-squared and $P$-values for allelic frequencies of SNP analyzed in the bovine $S P 110$ gene in infected and healthy animals of Spanish (HFS) and Dutch (HFN) Holstein-Friesian herds

\begin{tabular}{|c|c|c|c|c|c|c|c|c|c|c|}
\hline \multirow[b]{2}{*}{ SNP } & \multicolumn{5}{|c|}{ HFS } & \multicolumn{5}{|c|}{ HFN } \\
\hline & $\begin{array}{l}\text { Susceptibility } \\
\text { allele }\end{array}$ & $\begin{array}{l}\text { Frequencies: } \\
\text { case, control }\end{array}$ & $x^{2}$ & $\begin{array}{c}\text { Nominal } \\
P \text {-value }\end{array}$ & $\begin{array}{c}\text { Corrected } \\
P \text {-value }\end{array}$ & $\begin{array}{l}\text { Susceptibility } \\
\text { allele }\end{array}$ & $\begin{array}{l}\text { Frequencies: } \\
\text { case, control }\end{array}$ & $x^{2}$ & $\begin{array}{c}\text { Nominal } \\
P \text {-value }\end{array}$ & $\begin{array}{c}\text { Corrected } \\
P \text {-value }\end{array}$ \\
\hline c. $-1974 \mathrm{C}>\mathrm{T}$ & $\mathrm{T}$ & $0.099,0.048$ & 6.211 & 0.013 & NS & $\mathrm{T}$ & $0.034,0.025$ & 0.556 & 0.456 & NS \\
\hline c. $575-88 \mathrm{~T}>\mathrm{G}$ & $\mathrm{T}$ & $0.718,0.703$ & 0.148 & 0.7 & NS & G & $0.276,0.244$ & 1.01 & 0.315 & NS \\
\hline c. $587 \mathrm{~A}>\mathrm{G}$ & $\mathrm{A}$ & $0.886,0.744$ & 17.852 & $2.4 \times 10^{-5}$ & $2 \times 10^{-4 *}$ & $\mathrm{~A}$ & $0.706,0.633$ & 4.673 & 0.031 & NS \\
\hline c. $1414+173 \mathrm{~A}>\mathrm{T}$ & $\mathrm{A}$ & $0.819,0.740$ & 4.674 & 0.031 & NS & $\mathrm{A}$ & $0.603,0.553$ & 1.901 & 0.168 & NS \\
\hline c. $1557+106 \mathrm{~T}>\mathrm{C}$ & $\mathrm{T}$ & $0.806,0.740$ & 3.482 & 0.062 & NS & $\mathrm{T}$ & $0.603,0.550$ & 2.18 & 0.14 & NS \\
\hline c. $1558-191 \mathrm{C}>\mathrm{T}$ & $\mathrm{C}$ & $0.803,0.733$ & 3.777 & 0.052 & NS & $\mathrm{C}$ & $0.603,0.551$ & 2.127 & 0.145 & NS \\
\hline c. $1673+117 \mathrm{~T}>\mathrm{C}$ & $\mathrm{T}$ & $0.805,0.726$ & 4.599 & 0.032 & NS & $\mathrm{T}$ & $0.591,0.540$ & 1.929 & 0.165 & NS \\
\hline c. $1769 \mathrm{C}>\mathrm{T}$ & $\mathrm{C}$ & $0.798,0.733$ & 3.265 & 0.071 & NS & $\mathrm{C}$ & $0.612,0.550$ & 3.04 & 0.081 & NS \\
\hline c. $1782+72 \mathrm{C}>\mathrm{T}$ & $\mathrm{T}$ & $0.004,0.002$ & 0.202 & 0.653 & NS & $\mathrm{T}$ & $0.003,0.002$ & 0.025 & 0.875 & NS \\
\hline c. $1782+261 \mathrm{~A}>\mathrm{C}$ & A & $0.897,0.889$ & 0.089 & 0.766 & NS & $\mathrm{C}$ & $0.124,0.116$ & 0.093 & 0.76 & NS \\
\hline c. $1782+402 \mathrm{~A}>\mathrm{T}$ & $\mathrm{A}$ & $0.797,0.720$ & 4.263 & 0.039 & NS & $\mathrm{A}$ & $0.598,0.546$ & 2.098 & 0.147 & NS \\
\hline
\end{tabular}

${ }^{1} P$-value corrections by $1,000,000$ permutations.

$* P<0.05$.

the first block was significantly more frequent in infected animals (corrected $P=0.002$; Table 2). Accordingly, the unique haplotype with the protective $\mathrm{G}$ allele (TG) was significantly associated with lesser infection susceptibility (corrected $P=3 \times 10^{-4}$ ). No significant genetic association was detected between haplotypes and MAP infection in HFN (Table 2); the risk haplotype TA maintained the same trend observed in HFS, although in HFN, it did not reach significance. The TG haplotype exhibited a very similar frequency in healthy and infected animals in the HFN population.

Allelic frequencies for SP110 were significantly different in HFS and HFN $(P<0.001$, after Bonferroni correction). Thus, formally, 2 populations cannot be grouped. Nevertheless, it is important to point out that when all HF individuals were considered together, the analyzed SNP continued to be in Hardy-Weinberg equilibrium for the resulting control population. Moreover, genetic association analysis continued to show significant values for both the c.587A $>$ G SNP (corrected $P$ $=0.0017)$ and the protective TG haplotype (corrected $P=0.0015)$.

\section{In Silico Study of the c.587A>G Polymorphism}

The SNP c.587A $>\mathrm{G}$ is located in exon 5 and is a nonsynonymous SNP that leads to an amino acid change from asparagine to serine at codon 196 (p.Asn196Ser). Alignment of the coding sequences of the SP110 gene from 6 mammals (human, chimpanzee, horse, cow, mouse, and rat) showed that the A allele is well conserved among these species (results not shown). However, alignment of the corresponding protein sequences with MULTALIN software revealed that asparagine is not a conserved residue at this position due to variations in the other 2 nucleotides that make up the codon corresponding to asparagine. Amino acid sequence analysis with ELM showed several functional sites harboring the Asn amino acid at position 196. Of these, the only functional motif that disappears when the Asn to Ser change occurs at position 196 is the N-glycosylation site between the amino acids 195 and 200. Regarding protein structure, no changes in the secondary structure of the SP110 protein associated with the p.Asn196Ser mutation were predicted by JUFO software, because both amino acids were predicted to form a loop.

\section{DISCUSSION}

The present work constitutes the first characterization of SP110 gene variability in Bos taurus. This gene was screened in $30 \mathrm{HF}$ animals, and 17 new SNP were detected by comparative sequencing. With the complementary strategy of SNP annotation from databases, a total of 23 SNP were compiled. Of these, 14 were genotyped in $2 \mathrm{HF}$ populations, and 11 of them turned out to be common SNP. The SNP in the latter set form 2 differentiated haplotype blocks along the SP110 gene. In one block, c.575-88T $>\mathrm{G}$ and c.587A $>\mathrm{G}$ are in linkage disequilibrium, constituting a block that covers a small region between intron 4 and exon 5 and for which 3 haplotypes were observed in HFS and HFN. The other block is made up of 9 SNP and includes the region of the gene situated between introns 11 and 14 . In this block, 9 haplotypes were found, with the 3 most frequent haplotypes being common to both the HFS and the HFN subpopulations. 
The second aim of this work was to perform a population-based genetic association study between those genetic polymorphisms and susceptibility to MAP infection. Our results support the hypothesis that the SP110 gene is involved in susceptibility to MAP infection in HF cattle. The major allele A of the nonsynonymous SNP c.587A $>\mathrm{G}$ appears to be significantly associated with a greater risk of MAP infection in one of the analyzed subpopulations (HFS). This would suggest a possible protective effect of the minor allele $\mathrm{G}$. Indeed, the genotypic association test pointed to an additive effect for this allele, because increasing the number of $\mathrm{G}$ alleles leads to the greatest protection in homozygous GG. In addition, haplotype analyses for
SNP c.575-88T $>\mathrm{G}$ and the linked c.587A $>\mathrm{G}$ point to $\mathrm{TA}$ as being a risk haplotype and reveal a protective effect for the TG haplotype, the only haplotype that bears the $\mathrm{G}$ allele of the c.587A $>\mathrm{G}$ SNP.

In HFN, the same tendency was observed regarding SNP c.587A $>$ G, but it was not statistically significant. This may be due to the existence of a different genetic background in the analyzed populations, especially taking into account that HFS and HFN subpopulations share the same linkage disequilibrium pattern, but in HFN the frequency of SNP is greater and the sample size is larger. This differential genetic background could be in part related to the use of genetically distinct sires. In any event, the absence of genetic association in the

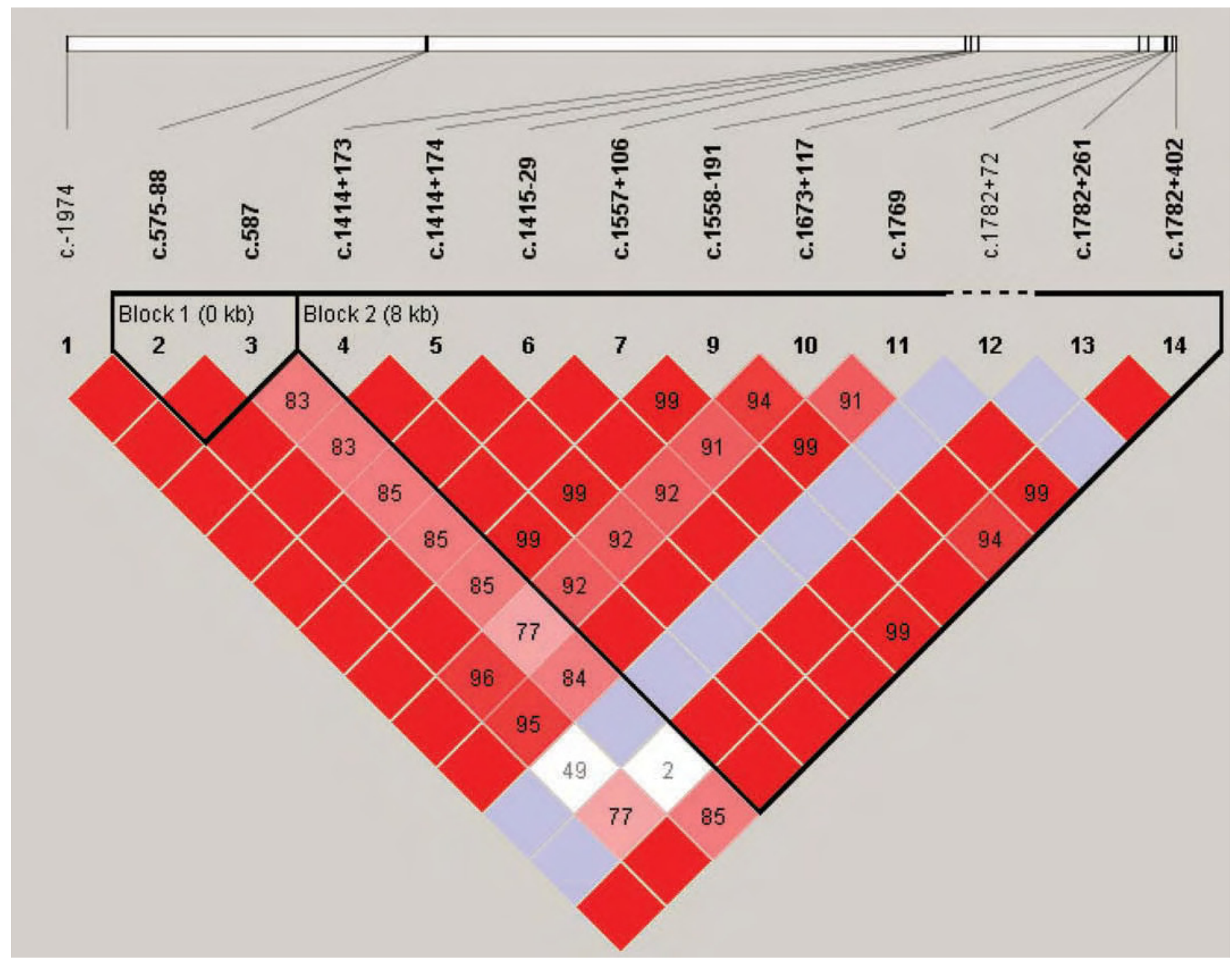

Figure 2. Linkage disequilibrium pattern and haplotype block structure for the bovine SP110 nuclear body protein gene in both analyzed Holstein-Friesian populations. The plot was generated using Haploview 4.1 (http://www.broadinstitute.org/mpg/haploview) with the D' Color Scheme $\left[\mathrm{D}^{\prime}=0,0<\mathrm{D}^{\prime}<1\right.$, and $\mathrm{D}^{\prime}=1$ are shown by white, pink (light gray), and red (dark gray), respectively], and pairwise $\mathrm{r}^{2}$ values are shown by diamonds $\left(\mathrm{r}^{2}=1\right.$ when no value is indicated $)$. Color version available in the online PDF. 
Table 2. Chi-squared and $P$-values for haplotype frequencies in the bovine SP110 gene in infected and healthy animals of Spanish (HFS) and Dutch (HFN) Holstein-Friesian herds

\begin{tabular}{|c|c|c|c|c|c|c|c|c|c|c|}
\hline \multirow[b]{2}{*}{ Haplotype } & \multicolumn{5}{|c|}{ HFS } & \multicolumn{5}{|c|}{ HFN } \\
\hline & Frequency & $\begin{array}{l}\text { Frequencies: } \\
\text { case, control }\end{array}$ & $\chi^{2}$ & $\begin{array}{l}\text { Nominal } \\
P \text {-value }\end{array}$ & $\begin{array}{c}\text { Corrected } \\
P \text {-value }\end{array}$ & Frequency & $\begin{array}{l}\text { Frequencies: } \\
\text { case, control }\end{array}$ & $x^{2}$ & $\begin{array}{c}\text { Nominal } \\
P \text {-value }\end{array}$ & $\begin{array}{c}\text { Corrected } \\
P \text {-value } \\
\end{array}$ \\
\hline \multicolumn{11}{|l|}{ Block 1} \\
\hline TA & 0.499 & $0.595,0.448$ & 12.783 & $3 \times 10^{-4}$ & $0.002^{*}$ & 0.409 & $0.432,0.390$ & 1.483 & 0.223 & NS \\
\hline GA & 0.293 & $0.285,0.297$ & 0.098 & 0.754 & NS & 0.334 & $0.293,0.366$ & 4.791 & 0.029 & NS \\
\hline TG & 0.208 & $0.120,0.255$ & 16.418 & $5 \times 10^{-5}$ & $3 \times 10^{-4 *}$ & 0.258 & $0.275,0.244$ & 0.982 & 0.322 & NS \\
\hline TAACTCTAT & 0.222 & $0.172,0.249$ & 5.076 & 0.024 & NS & 0.416 & $0.388,0.439$ & 2.048 & 0.152 & NS \\
\hline ATGTCTCCA & 0.105 & $0.099,0.108$ & 0.124 & 0.725 & NS & 0.119 & $0.120,0.117$ & 0.014 & 0.907 & NS \\
\hline ATGTTCCAT & 0.013 & $0.010,0.014$ & 0.184 & 0.668 & NS & 0.008 & $0.006,0.009$ & 0.289 & 0.591 & NS \\
\hline TAACTTTAT & 0.012 & $0.015,0.011$ & 0.246 & 0.62 & NS & 0.008 & $0.006,0.009$ & 0.253 & 0.615 & NS \\
\hline ATGTCCCAA & 0.01 & $0.015,0.007$ & 1.107 & 0.293 & NS & 0.007 & $0.009,0.005$ & 0.536 & 0.46 & NS \\
\hline ATGTCCCCA & 0.004 & $0.007,0.002$ & 1.046 & 0.306 & NS & 0 & $0.000,0.000$ & $\mathrm{NA}^{2}$ & NA & NA \\
\hline ATGTCTTAA & 0.002 & $0.000,0.002$ & 0.499 & 0.48 & NS & 0 & $0.000,0.000$ & NA & NA & NA \\
\hline
\end{tabular}

${ }^{1} P$-value corrections by $1,000,000$ permutations.

${ }^{2} \mathrm{NA}=$ not analyzed.

$* P<0.05$.

HFN population raises the need to analyze new independent populations, with an aim of testing the repeatability of the results obtained in the HFS population.

Information regarding polymorphisms in genes that are orthologs of the bovine $S P 110$ gene and their effects in the corresponding proteins is very scarce. Indeed, the possible association between SP110 gene polymorphisms and mycobacterial infections has been studied only in the human species. Tosh et al. (2006) detected 3 SNP associated with susceptibility to tuberculosis in West Africa populations; 2 of these were intronic and the third was a nonsynonymous SNP localized in exon 11. As in the present study, this coding SNP was not localized to any of the principal domains of the SP110 protein, and the authors did not establish a possible functional effect of this SNP, suggesting possible linkage disequilibrium between this SNP and the real causal variant. In any event, remember that the results reported by Tosh et al. (2006) have not been confirmed in subsequent studies (Thye et al., 2006; Babb et al., 2007; Szeszko et al., 2007).

Returning to the results of the present study, the coding SNP c.587A $>\mathrm{G}$, associated with susceptibility, is located in exon 5 of the bovine SP110 gene, corresponding to a region between the Sp100 and SAND domains. The function of these domains is not entirely clear, although the Sp100 domain has been reported as a potential mediator of homodimer formation (Seeler et al., 1998) and the chromatin-binding SAND domain is most likely involved in transcriptional regulation (Bottomley et al., 2001). The A to $\mathrm{G}$ nucleotide mutation results in an amino acid change in the codon 196 position of the predicted protein (p.Asn196Ser). The A allele, associated in the present study with susceptibility to MAP infection, results in an asparagine (AAC codon), whereas the protective $\mathrm{G}$ allele results in a serine (AGC codon). The paucity of information regarding this gene in other mammalian species substantially limits in silico analysis. Nevertheless, sequence alignment analysis suggests that the A allele is a conserved nucleotide, although at the protein level, Bos taurus and Equus caballus are the only species that present an asparagine in this position, indicating that the asparagine amino acid at position 196 is not a conserved residue. On the other hand, both asparagine and serine amino acids were predicted to form a loop in the secondary structure of the SP110 protein, implying that the SNP c.587A $>\mathrm{G}$ would not involve changes at this level. However, a potential Nglycosylation site involving asparagine at position 196 was found, which would be disrupted by the presence of a serine at the same position. Asparagine glycosylation is a common posttranslational modification implicated in different cellular processes, such as protein folding, protein secretion, intracellular trafficking, and cell communication (Skropeta, 2009), and researchers have reported that mutations in these functional sites can have a direct effect on the stability, assembly, and function of the affected proteins (Ramanathan and Hall, 1999; Li and Pajor, 2003; Meng et al., 2007). On the basis of the present results, it is conceivable that the disruption of the putative N-glycosylation site in the bovine SP110 protein could modify, in some way, its transcriptional 
coactivator function and, consequently, alter the fate of MAP in macrophages.

\section{CONCLUSIONS}

Results obtained substantiate the hypothesis that variants of bovine SP110, in combination with other risk alleles from different genes and interacting with environmental factors, contribute to the outcome of MAP infection in cattle. The SNP c.587A $>$ G in exon 5 showed a significant association with susceptibility to MAP infection. Functional analyses will be required to understand the exact mechanism by which this polymorphism controls MAP within the macrophage; however, a modification of an N-glycosylation site caused by this SNP has been predicted, which a priori, could influence protein function. Even so, the possible functionality of those intronic SNP that could not be genotyped cannot be ruled out. Furthermore, other significant causal variants could be localized in some of the regions of the SP110 gene that have not yet been sequenced. The reporting of new SNP and analysis of their putative association with MAP infection could shed more light on this matter. Despite the necessity of repeating this investigation in other independent studies, the identification of the bovine $S P 110$ gene as a susceptibility gene for Johne's disease, one of the most important diseases in dairy cattle industry, deepens our understanding of the molecular basis of this disease and constitutes a further step toward the implementation of marker-assisted selection in breeding programs aimed at controlling paratuberculosis.

\section{ACKNOWLEDGMENTS}

Technical and human support provided by SGIker (UPV/EHU, MICINN, GV/EJ, ESF) is gratefully acknowledged. The authors thank also Fernando Rendo (University of the Basque Country, Leioa, Spain) for technical assistance. This study was funded by the Spanish Ministry of Education and Science (MEC, AGL2006-14315-C02-02/GAN), the University of the Basque Country (Leioa, Spain), Bizkaiberri program of Biscay Regional Government, Aberekin Insemination Centre, and the Agriculture and Fishing Department of the Basque Government (Derio, Spain).

\section{REFERENCES}

Applied Biosystems. 2006. Custom TaqMan Genomic Assays: Protocol, Submission Guidelines. http://www.appliedbiosystems.com

Babb, C., E. H. Keet, P. D. van Helden, and E. G. Hoal. 2007. SP110 polymorphisms are not associated with pulmonary tuberculosis in a South African population. Hum. Genet. 121:521-522.
Barrett, J. C., B. Fry, J. Maller, and M. J. Daly. 2005. Haploview: Analysis and visualization of LD and haplotype maps. Bioinformatics 21:263-265.

Bloch, D. B., A. Nakajima, T. Gulick, J. D. Chiche, D. Orth, S. M. de la Monte, and K. D. Bloch. 2000. Sp110 localizes to the PMLSp100 nuclear body and may function as a nuclear hormone receptor transcriptional coactivator. Mol. Cell. Biol. 20:6138-6146.

Bottomley, M. J., M. W. Collard, J. I. Huggenvik, Z. Liu, T. J. Gibson, and M. Sattler. 2001. The SAND domain structure defines a novel DNA-binding fold in transcriptional regulation. Nat. Struct. Biol. 8:626-633.

Camargo, A., F. Azuaje, H. Wang, and H. Zheng. 2008. Permutationbased statistical tests for multiple hypotheses. Source Code Biol. Med. 3:15.

Corpet, F. 1988. Multiple sequence alignment with hierarchical clustering. Nucleic Acids Res. 16:10881-10890.

Gabriel, S. B., S. F. Schaffner, H. Nguyen, J. M. Moore, J. Roy, B. Blumenstiel, J. Higgins, M. DeFelice, A. Lochner, M. Faggart, S. N. Liu-Cordero, C. Rotimi, A. Adeyemo, R. Cooper, R. Ward, E. S. Lander, M. J. Daly, and D. Altshuler. 2002. The structure of haplotype blocks in the human genome. Science 296:2225-2229.

González, J. R., L. Armengol, X. Solé, E. Guinó, J. M. Mercader, X. Estivill, and V. Moreno. 2007. SNPassoc: An R package to perform whole genome association studies. Bioinformatics 23:644-645.

Hawken, R. J., W. C. Barris, S. M. McWilliam, and B. P. Dalrymple. 2004. An interactive bovine in silico SNP database (IBISS). Mamm. Genome 15:819-827.

Juliarena, M. A., M. Poli, L. Sala, C. Ceriani, S. Gutierrez, G. Dolcini, E. M. Rodríguez, B. Mariño, C. Rodríguez-Dubra, and E. N. Esteban. 2008. Association of BLV infection profiles with alleles of the BoLADRB3.2 gene. Anim. Genet. 39:432-438.

Koets, A., W. Santema, H. Mertens, D. Oostenrijk, M. Keestra, M. Overdijk, R. Labouriau, P. Franken, A. Frijters, M. Nielen, and V. Rutten. 2010. Susceptibility to paratuberculosis infection in cattle is associated with single nucleotide polymorphisms in Toll-like receptor 2 which modulate immune responses against Mycobacterium avium subspecies paratuberculosis. Prev. Vet. Med. 93:305-315.

Li, H., and A. M. Pajor. 2003. Mutagenesis of the N-glycosylation site of hNaSi-1 reduces transport activity. Am. J. Physiol. Cell Physiol. 285:C1188-C1196.

Meng, J. F., C. McFall, and L. J. Rosenwasser. 2007. Polymorphism $\mathrm{R} 62 \mathrm{~W}$ results in resistance of CD23 to enzymatic cleavage in cultured cells. Genes Immun. 8:215-223.

Mucha, R., M. R. Bhide, E. B. Chakurkar, M. Novak, and I. Mikula. 2009. Toll-like receptors TLR1, TLR2 and TLR4 gene mutations and natural resistance to Mycobacterium avium ssp. paratuberculosis infection in cattle. Vet. Immunol. Immunopathol. 128:381388.

Pan, H., B. S. Yan, M. Rojas, Y. V. Shebzukhov, H. Zhou, L. Kobzik, D. E. Higgins, M. J. Daly, B. R. Bloom, and I. Kramnik. 2005. Ipr1 gene mediates immunity to tuberculosis. Nature 434:767-772.

Pinedo, P. J., C. D. Buergelt, G. A. Donovan, P. Melendez, L. Morel, R. Wu, T. Y. Langaee, and D. O. Rae. 2009a. Association between CARD15/NOD2 gene polymorphisms and paratuberculosis infection in cattle. Vet. Microbiol. 134:346-352.

Pinedo, P. J., C. D. Buergelt, G. A. Donovan, P. Melendez, L. Morel, R. Wu, T. Y. Langaee, and D. O. Rae. 2009b. Candidate gene polymorphisms (BoIFNG, TLR 4, SLC11A1) as risk factors for paratuberculosis infection in cattle. Prev. Vet. Med. 91:189-196.

R Development Core Team. 2008. R: A Language and Environment for Statistical Computing. R Foundation for Statistical Computing, Vienna, Austria. http://www.R-project.org

Ramanathan, V. K., and Z. W. Hall. 1999. Altered glycosylation sites of the delta subunit of the acetylcholine receptor $(\mathrm{AChR})$ reduce alpha delta association and receptor assembly. J. Biol. Chem. 274:20513-20520.

Rousset, F. 2008. Genepop'007: A complete reimplementation of the GENEPOP software for Windows and Linux. Mol. Ecol. Resour. 8:103-106.

Ruiz-Larrañaga, O., J. M. Garrido, M. Iriondo, C. Manzano, E. Molina, A. P. Koets, V. P. M. G. Rutten, R. A. Juste, and A. Es- 
tonba. 2010b. Genetic association between bovine NOD2 polymorphisms and infection by Mycobacterium avium ssp. paratuberculosis in Holstein-Friesian cattle. Anim. Genet. doi:10.1111/j.13652052.2010.02055.x.

Ruiz-Larrañaga, O., J. M. Garrido, C. Manzano, M. Iriondo, E. Molina, A. Gil, A. P. Koets, V. P. M. G. Rutten, R. A. Juste, and A. Estonba. 2010a. Identification of single nucleotide polymorphisms in the bovine solute carrier family 11 member 1 (SLC11A1) gene and their association with infection by Mycobacterium avium subspecies paratuberculosis. J. Dairy Sci. 93:1713-1721.

Schulman, N. F., S. M. Víitala, D. J. de Koning, J. Virta, A. MäkiTanila, and J. H. Vilkki. 2004. Quantitative trait loci for health traits in Finnish Ayrshire cattle. J. Dairy Sci. 87:443-449.

Seeler, J. S., A. Marchia, D. Sitterlin, C. Transy, and A. Dejean. 1998. Interaction of Sp100 and HP1 proteins: A link between the promyelocytic leukemia-associated nuclear bodies and the chromatin compartment. Proc. Natl. Acad. Sci. USA 95:7316-7321.

Sharma, B. S., I. Leyva, F. Schenkel, and N. Karrow. 2006. Association of toll-like receptor 4 polymorphisms with somatic cell score and lactation persistency in Holstein bulls. J. Dairy Sci. 89:36263635.

Skropeta, D. 2009. The effect of individual N-glycans on enzyme activity. Bioorg. Med. Chem. 17:2645-2653.

Szeszko, J., B. Healy, H. Stevens, Y. Balabanova, F. Drobniewski, J. A. Todd, and S. Nejentsev. 2007. Resequencing and association analysis of the SP110 gene in adult pulmonary tuberculosis. Hum. Genet. 121:155-160.

Thye, T., E. N. Browne, M. A. Chinbuah, J. Gyapong, I. Osei, E. Owusu-Dabo, S. Niemann, S. Rusch-Gerdes, R. D. Horstmann, and C. G. Meyer. 2006. No associations of human pulmonary tuberculosis with Sp110 variants. J. Med. Genet. 43:e32.

Tosh, K., S. J. Campbell, K. Fielding, J. Sillah, B. Bah, P. Gustafson, K. Manneh, I. Lisse, G. Sirugo, S. Bennett, P. Aaby, K. P. McAdam, O. Bah-Sow, C. Lienhardt, I. Kramnik, and A. V. Hill 2006. Variants in the SP110 gene are associated with genetic susceptibility to tuberculosis in West Africa. Proc. Natl. Acad. Sci USA 103:10364-10368. 